\title{
Redistribuição de nutrientes em folhas de pinhão-manso entre estádios fenológicos
}

\author{
Rosiane. L. S. de Lima', Liv S. Severino ${ }^{2}$, Jairo 0. Cazetta ${ }^{3}$, \\ Carlos A. V. de Azevedo ${ }^{4}$, Valdinei Sofiatti $\&$ Nair H. C. Arriel ${ }^{5}$
}

RESU M 0

A redistribuição dos nutrientes minerais das folhas maduras para as estruturas em crescimento é um importante processo na fisiologia das plantas. Cada nutriente é redistribuído em proporções e fases variadas. O bjetivou-se, com este trabalho, quantificar a redistribuição de macro e micronutrientes em folhas de pinhão-manso (Jatropha curcas) em função do estádio fenológico. Portanto, os tratamentos se constituíram em cinco estádios fenológicos da folha (folha em crescimento, folha jovem, folha madura, folha senescente e folhedo) com cinco repetições. O s nutrientes $P, K, C u$ e $Z n$ são intensamente redistribuídos das folhas mais velhas para os tecidos mais jovens da planta de pinhão-manso enquanto há pouca redistribuição de $\mathrm{N}, \mathrm{Ca}, \mathrm{Mg}$, Fe e $\mathrm{Mn}$. Com a senescência das folhas os nutrientes que não se redistribuem representam perda para a planta visto que precisam ser reabsorvidos para suportar 0 crescimento de novas estruturas.

Palavras-chave: Jatropha curcas, translocação de nutrientes, nutrição

\section{Redistribution of nutrients in jatropha leaves through phenological phases}

\begin{abstract}
N utrient redistribution from mature leaves to growing structures is an important process in plant physiology. Each nutrient is redistributed in a different rate and time. This study aimed to quantify redistribution of macronutriens and minor nutrients in Jatropha curcas leaves through five phenological phases (expanding leaf, young leaf, mature leaf, senescing leaf, and dry leaf). It was observed that the nutrients $\mathrm{P}, \mathrm{K}, \mathrm{Cu}$, and $\mathrm{Zn}$ are intensively redistributed from older to younger jatropha leaves, while $\mathrm{N}, \mathrm{Ca}, \mathrm{Mg}, \mathrm{Fe}$, and $\mathrm{Mn}$ are poorly redistributed. Leaf senescence causes a loss of the nutrients that are not redistributed because they will need to be re-absorbed for supporting the growth of new structures in the plant.
\end{abstract}

Key words: Jatropha curcas, nutrients translocation, nutrition

\footnotetext{
${ }^{1}$ Pesquisadora DCR pela FAPESQ/PB/CN Pq. Rua O svaldo Cruz 1143, Centenário, CEP. 58107-720, Campina Grande, PB, Fone: (83) $3182-4300$. E-mail: limarosiane@yahoo.com.br

2 D outorando em Agronomia pela Texas Tech University, Lubbock, TX, USA. Embrapa Algodão. E-mail: liv@cnpa.embrapa.br

3 Departamento de Tecnologia. U NESP. CEP: 14.884-900, Jaboticabal, SP. Fone: (16) 3209-2675. E-mail: cazetta@fcav.unesp.br

${ }^{4}$ U AEA. Rua Aprígio Veloso 882, Bodocongó, CEP. 58429-140. Campina Grande, PB. Fone: (83) 3333-1056. E-mail: cazevedo@deag.ufcg.edu.br ${ }^{5}$ Embrapa Algodão. Rua 0 svaldo Cruz 1143, Centenário, CEP. 58.107-720, Campina Grande, Fone: (83) 318243-00. E-mail: vsofiatti@cnpa.embrapa.br; nair@cnpa.embrapa.br
} 


\section{INTRODUÇÃO}

O pinhão-manso (Jatropha curcas L.) é uma espécie perene pertencente à família das Euforbiáceas, de porte arbustivo e crescimento rápido, originária da América Central mas se encontra vegetando espontaneamente em diversas regiões do Brasil (Laviola \& Dias, 2008). Seu cultivo despertou o interesse econômico após a descoberta do alto teor de óleo apresentado em suas sementes (33 a 38\%) o que representa entre 53 e $79 \%$ do peso de seus frutos e por se tratar de uma planta rústica adaptada a diversas condições edafoclimáticas (Arruda et al., 2004; Lima et al., 2010; 2011).

Por se tratar de uma planta caducifólia, esta oleaginosa apresenta grande potencial de reciclagem de nutrientes por permitir que grande quantidade de material vegetal retorne ao solo pela queda do folhedo.

A redistribuição (mobilidade ou remobilização) dos nutrientes minerais das folhas maduras para as áreas de crescimento é essencial para a conclusão do ciclo de vida das plantas (Camargos \& Muraoka, 2007). Entretanto, a quantidade redistribuída difere entre nutrientes minerais e reflete na ocorrência de sintomas visuais de deficiência nutricional. De acordo com Marschner (2002), o aparecimento de sintomas de deficiência nas folhas mais velhas reflete a alta taxa de redistribuição do nutriente. Por outro lado, o surgimento de sintomas nas folhas mais novas e de meristemas apicais reflete uma redistribuição insuficiente em virtude da redistribuição se dar, predominantemente, pelo floema (Marschner, 2002).

A dinâmica de nutrientes em espécies perenes varia em função da espécie, da idade da planta, do estádio fenológico, das condições edafoclimáticas e das práticas de manejo adotadas. De acordo com George et al. (1989), ao longo do ciclo a concentração de alguns elementos nas folhas aumenta enquanto a de outros decresce, ocorrendo translocação de nutrientes de órgãos senescentes para regiões de crescimento das plantas, como folhas novas e estruturas reprodutivas.

Para Kumar \& Pandey (1979), os elementos de alta mobilidade no floema, tais como N, P e K, se apresentaram em maiores quantidades nas folhas mais jovens. Entretanto, nutrientes como $\mathrm{Ca}$ e $\mathrm{Mg}$ tendem a se concentrar em tecidos mais velhos visto que possui baixa capacidade de redistribuição (Pathar \& Pandey, 1976; Caldeira et al., 2002). A elevação dos teores de cálcio com o envelhecimento das folhas pode ser explicada sobretudo pelo aumento na produção de material estrutural na massa seca e de compostos de reserva e, ainda, pela baixa mobilidade deste elemento no floema (Marschner, 2002).

Objetivou-se, com este trabalho, quantificar a redistribuição de macro e micronutrientes em folhas de pinhão-manso entre diferentes estádios fenológicos.

\section{Material E MÉTODOS}

Plantas de pinhão-manso com 3,5 anos de idade foram selecionadas em uma lavoura comercial situada na Fazenda Estivas, município de Garanhuns, Estado de Pernambuco $\left(8^{\circ}\right.$ 56' 30" de latitude Sul, 36 27' 97' de longitude Oeste e 741 m de altitude). A precipitação média anual da região é de $788 \mathrm{~mm}$ (ITEP, 2008), clima BS (seco, semiárido, megatérmico, com oito meses de estiagem) segundo a classificação de Köppen. Coletaram-se amostras de solo entre fileiras, na área de cultivo, cujas características químicas se encontram na Tabela 1.

Adotou-se um delineamento inteiramente casualizado com cinco tratamentos, cinco repetições e uma planta por parcela. Os tratamentos se constituíram de cinco estádios fenológicos da folha (folha em crescimento, folha jovem, folha madura, folha senescente e folhedo). As amostras de folhas foram coletadas no início da estação chuvosa, em cinco arbustos de pinhãomanso, escolhidos aleatoriamente. Em cada unidade experimental foram colhidas 4 folhas, totalizando 20 folhas por amostra composta. Colheram-se folhas em quatro estádios fenológicos (em crescimento, jovem, maduras e senescentes) na parte mediana da copa das plantas nos quatro pontos ortogonais, sendo colhida uma folha em cada ponto cardeal. Os folhedos foram folhas caídas há pouco tempo, ainda sem indício de decomposição, coletadas na projeção da copa.

O estádio fenológico das folhas foi determinado pela observação da cor, tamanho e posição no ramo. Folhas em crescimento eram menores que as folhas maduras (aproximadamente 2/3 do tamanho médio das folhas) e posicionadas na extremidade distal do ramo. Folhas jovens já estavam completamente expandidas (tamanho normal), estando posicionadas após as folhas em crescimento. Folhas maduras são aquelas posicionadas na parte mediana ou basal do ramo, de cor verde-escuro. As folhas senescentes se encontravam na base do ramo e tinham cor amarelada.

As folhas coletadas foram lavadas para retirada de impurezas, secadas em estufa de circulação de ar forçada, moídas e enviadas ao Laboratório de Análises de Solo e Nutrição de Plantas da Universidade Estadual Paulista, Campus de Jaboticabal, onde foram submetidas às análises químicas. Subamostras trituradas foram submetidas a digestão nítricoperclórica para a determinação das concentrações de P, K, Ca, $\mathrm{Mg}$ e $\mathrm{S}, \mathrm{Cu}, \mathrm{Fe}, \mathrm{Mn}$ e $\mathrm{Zn}$, e a digestão sulfúrica, para determinação do teor de $\mathrm{N}$. O N foi quantificado pelo método colorimétrico de Nessler; o $\mathrm{P}$ pelo método do fosfomolibdato e a redução pela vitamina $\mathrm{C}$, modificada por Braga \& Defelipo (1974); o K, por fotometria de chama, o S por turbidimetria do sulfato de bário (Blanchar et al., 1963) e os demais (Ca, Mg, Cu, $\mathrm{Fe}, \mathrm{Mn}$ e $\mathrm{Zn}$ ) foram quantificados por espectrofotometria de absorção atômica em chama.

Tabela 1. Resultados da análise de fertilidade do solo da área cultivada com pinhão-manso

\begin{tabular}{|c|c|c|c|c|c|c|c|c|c|c|c|}
\hline \multirow{2}{*}{$\underset{(1: 2,5)}{\mathrm{pH}}$} & $\mathrm{Ca}^{2+}$ & $\mathrm{Mg}^{2+}$ & $\mathrm{Na}^{+}$ & $\mathrm{K}^{+}$ & $\mathbf{S}$ & $\mathrm{H}+\mathrm{Al}$ & $T$ & \multirow{2}{*}{$\begin{array}{c}V \\
(\%)\end{array}$} & \multirow{2}{*}{$\begin{array}{c}\mathrm{Al}^{3+} \\
\left(\mathrm{mmol}_{\mathrm{c}} \mathrm{dm}^{-3}\right)\end{array}$} & \multirow{2}{*}{$\begin{array}{c}P \\
\left(\mathrm{mg} \mathrm{dm}^{-3}\right)\end{array}$} & \multirow{2}{*}{$\begin{array}{l}M .0 \\
\mathrm{~g} \mathrm{~kg}\end{array}$} \\
\hline & \multicolumn{7}{|c|}{ Complexo sortivo $\left(\mathrm{mmol}_{\mathrm{c}} \mathrm{dm}^{-3}\right)$} & & & & \\
\hline 6,2 & 25,7 & 14,3 & 1,47 & 2,3 & 43,7 & 14,0 & 57 & 76 & 0,5 & 11,4 & 14,7 \\
\hline
\end{tabular}


Os dados obtidos foram submetidos à análise de variância e ao teste $\mathrm{F}(5 \%)$ e as médias comparadas entre si pelo teste de Tukey (5\%), segundo recomendações de Santos \& Gheyi (2003).

\section{RESULTADOS E DISCUSSÃO}

O resumo da análise de variância é apresentado na Tabela 2, em que os teores foliares de todos os macro e micronutrientes tiveram variações significativas entre as fases fenológicas.

Os teores foliares de $\mathrm{N}$ foram mais altos nas folhas em crescimento $\left(43,5 \mathrm{~g} \mathrm{~kg}^{-1}\right)$ e diminuíram gradualmente entre cada estádio fenológico, obtendo-se o valor mais baixo no folhedo $\left(41,8 \mathrm{~g} \mathrm{~kg}^{-1}\right)$ (Figura 1A). O nitrogênio tem alta mobilidade no floema (Marschner, 2002) e se espera que seu teor seja menor nas folhas senescentes já que este nutriente pode ser redistribuído para outros órgãos, antes da queda da folha. No entanto, a redução no teor de $\mathrm{N}$ entre folhas jovens e senescentes, embora estatisticamente significativa, é relativamente pequena e proporciona pouca redistribuição do nutriente, ou seja, a maior parte do $\mathrm{N}$ ao invés de estar sendo redistribuída na planta foi perdida na queda da folha. Resultados similares aos observados neste estudo foram constatados por Medeiros \& Haridasan (1985), por Leitão \& Silva (2004) e por Camargos \& Muraoka (2007), ao constatarem que o $\mathrm{N}$ é redistribuído das folhas senescentes para os drenos.

$\mathrm{O}$ teor de $\mathrm{P}$ foi muito alto nas folhas em crescimento $(3,2$ $\mathrm{g} \mathrm{kg}^{-1}$ ) e apresentou redução a cada novo estádio fenológico, atingindo o valor de $\left(2,0 \mathrm{~g} \mathrm{~kg}^{-1}\right)$ no folhedo (Figura 1B). O P tem grande mobilidade no floema e é um nutriente muito ligado aos processos fisiológicos de fluxo energético (Marschner, 2002). Como o crescimento das folhas demanda grande quantidade de energia é provável que este nutriente seja translocado para a folha nesta fase, mas posteriormente a demanda seja reduzida

Tabela 2. Resumo da análise de variância dos teores de macro e micronutrientes em folhas de pinhão-manso em diferentes estádios fenológicos

\begin{tabular}{|c|c|c|c|c|c|c|c|c|c|c|c|}
\hline \multirow{2}{*}{ F. V. } & \multirow{2}{*}{ G. I. } & \multicolumn{10}{|c|}{ Quadrados médios } \\
\hline & & N & $\mathbf{P}$ & K & $\mathrm{Ca}$ & Mg & $S$ & $\mathrm{Cu}$ & $\mathrm{Fe}$ & Mn & $\mathrm{Zn}$ \\
\hline Tratamento & 4 & $6,52 *$ & $13,59 *$ & $412,3 *$ & $1449,1^{*}$ & $18,6^{*}$ & $1,97 *$ & $74,3^{*}$ & $38321,3^{*}$ & $2349,9 *$ & $896,2^{*}$ \\
\hline Resíduo & 12 & 0,49 & 0,20 & 11,2 & 21,7 & 1,5 & 0,015 & 1,2 & 1824,2 & 75,8 & 5,6 \\
\hline CV (\%) & - & 1,67 & 14,9 & 14,7 & 18,2 & 19,5 & 7,77 & 12,4 & 27,9 & 16,3 & 9,7 \\
\hline
\end{tabular}

A.

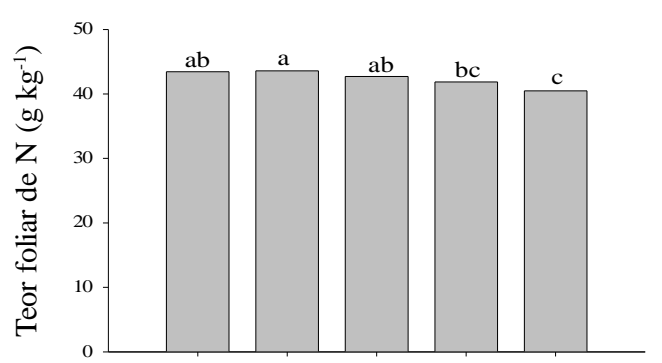

C.

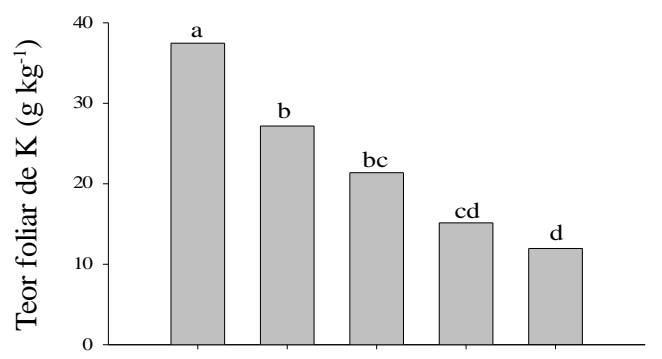

E

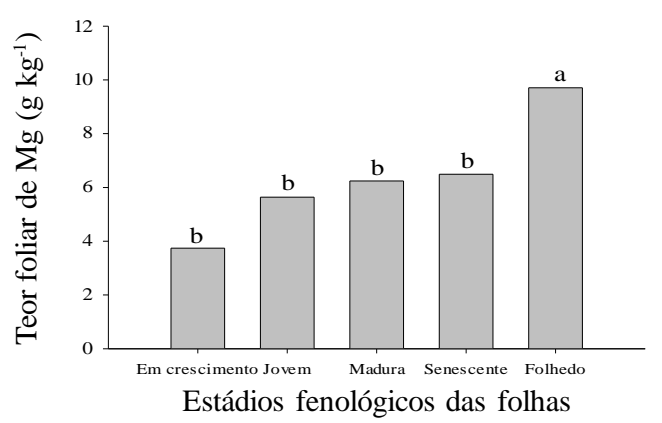

B.

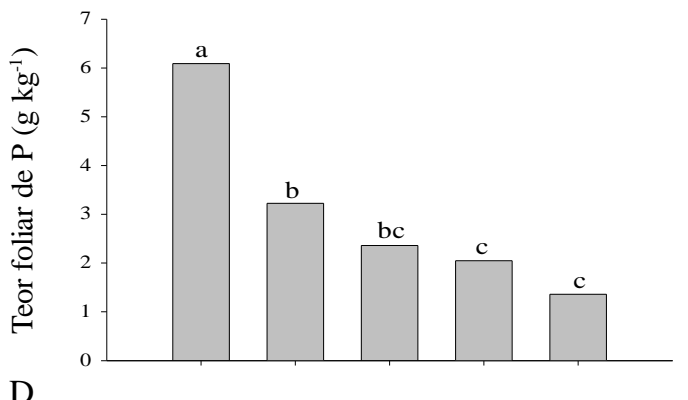

D.

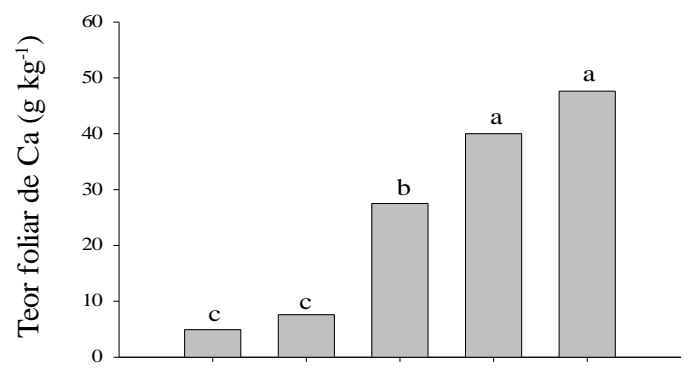

F.

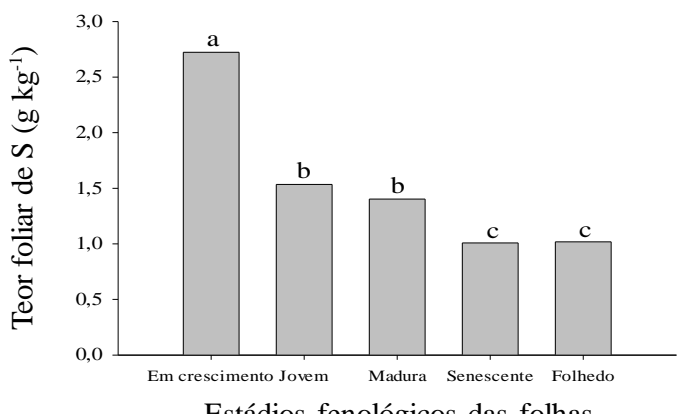

Estádios fenológicos das folhas

Figura 1. Teores de N (A), P (B), K (C), Ca (D), M g (E) e S (F), em folhas de pinhão-manso em diferentes estádios fenológicos 
e, aproveitando sua alta mobilidade, ele seja redistribuído para outras partes da planta em que a demanda esteja maior. Referidos resultados são similares aos constatados por Kumar \& Pandey (1979) em goiabeiras.

Os teores de $\mathrm{K}$ foram altos nas folhas em crescimento e reduziram com o avanço das fases fenológicas de forma similar ao $\mathrm{P}$, mas com menor intensidade (Figura 1C). O K também tem alta mobilidade no floema (Marcschner, 2002). A maior parte do potássio é absorvida pelas plantas durante a fase de crescimento vegetativo, pois este tem papel fundamental na fotossíntese e síntese de carboidratos (Yamada, 1987) e ativação de enzimas para diversos processos fisiológicos. Os teores são mais altos nas folhas jovens nas quais a atividade fisiológica é mais intensa (como fotossíntese) mas, à medida em que esses processos sofrem redução, o K é redistribuído para outras partes da planta. Por ter sido redistribuído, a perda de K pela queda das folhas é reduzida. Villela \& Lacerda (1992), estudando as espécies de cerrado Vochysia rufa e Curatella americana, também constataram teores mais elevados de potássio nas folhas jovens sugerindo a reabsorção deste elemento, antes da abscisão.

O cálcio tem baixa mobilidade na planta razão pela qual se acumula nas folhas, sem poder ser redistribuído para outras partes da planta antes da queda das folhas (Epstein \& Bloom, 2006; Marschner, 2002). O teor deste nutriente foi de 4,9 $\mathrm{g} \mathrm{kg}^{-1}$ nas folhas jovens e de 40,0 $\mathrm{g} \mathrm{kg}^{-1}$ no folhedo (Figura 1D). A insolubilidade dos compostos de cálcio da planta e sua localização na célula explicam, em parte, a limitada redistribuição (Malavolta, 2006).

O magnésio, embora seja considerado um nutriente móvel (Marschner, 2002), comportou-se de maneira similar ao Ca, apresentando teores mais elevados nas folhas mais velhas (Figura 1E). Segundo Malavolta (2006), é comum encontrar maior concentração de magnésio em folhas mais velhas do que em folhas jovens.

Embora o enxofre seja considerado um nutriente de pouca mobilidade (Marschner, 2002), observaram-se teores elevados nas folhas jovens e, ao invés de acúmulo, uma pequena redução no teor nas folhas mais velhas (Figura 1F). A redução do teor entre as folhas jovens e maduras $\left(2,7 \mathrm{e} 1,4 \mathrm{~g} \mathrm{~kg}^{-1}\right)$ para as senescentes e folhedo $\left(1,1 \mathrm{e} 1,0 \mathrm{~g} \mathrm{~kg}^{-1}\right)$ não foi muito intensa sendo compatível com a baixa mobilidade do nutriente; no entanto, o teor mais elevado nas folhas em crescimento é atípico e pode ter sido provocado por um acúmulo temporário que seria diluído após o crescimento da folha ou por uma variação ocasional devida a fatores ambientais.

O cobre e o zinco são nutrientes de baixa mobilidade (Marschner, 2002; Epstein \& Bloom, 2006) que também apresentaram altos teores nas folhas em crescimento mais variações ocasionais e sem tendência definida nos demais estádios fisiológicos (Figura 2A e D). O teor de zinco pode sofrer variações sazonais (Amaral et al., 2002), fator que pode influenciar parte da variação observada entre os estádios fenológicos da planta. Pelo teor observado no folhedo os nutrientes $\mathrm{Cu}$ e $\mathrm{Zn}$ não são redistribuídos na planta antes da senescência foliar.
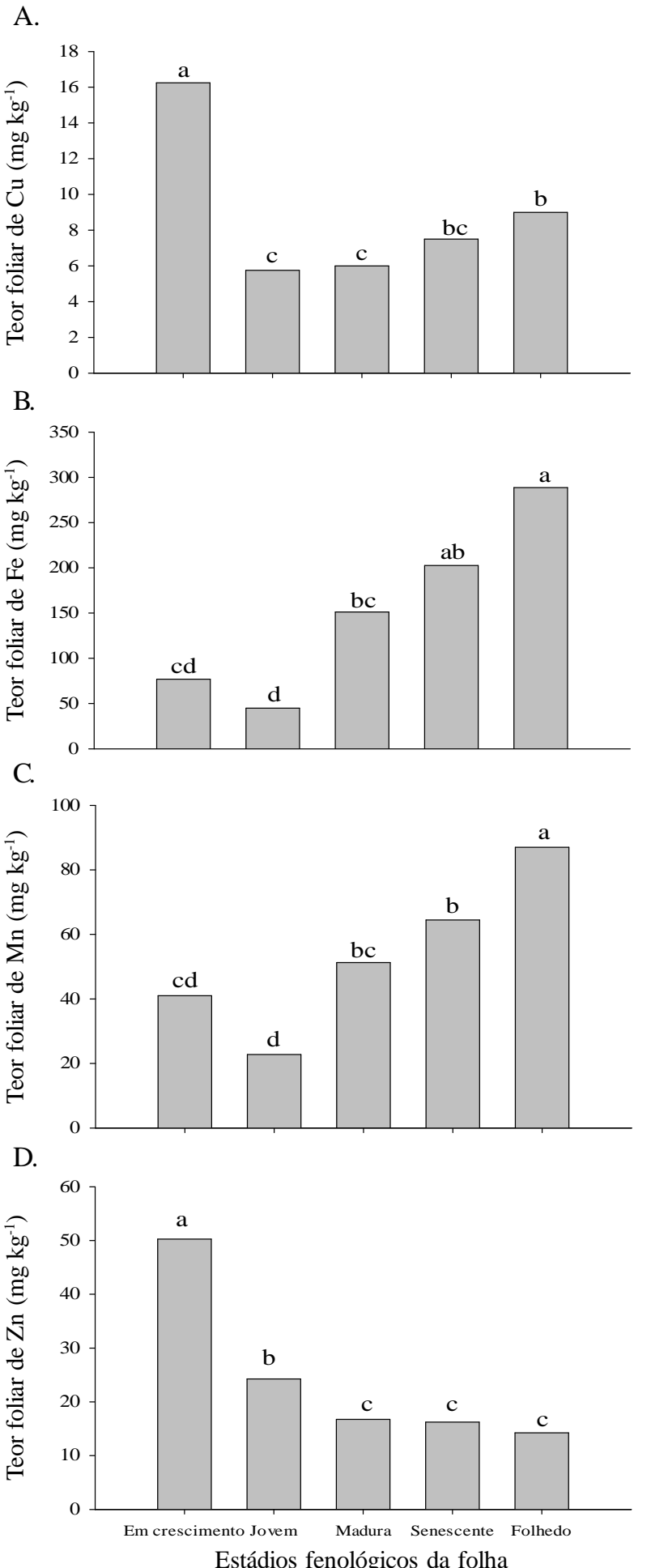

Figura 2. Teores de $\mathrm{Cu}(\mathrm{A}), \mathrm{Fe}(\mathrm{B}), \mathrm{Mn}(\mathrm{C})$ e $\mathrm{Zn}(\mathrm{D})$, em folhas de pinhão-manso colhidas em diferentes estádios fenológicos

O ferro e o manganês apresentaram comportamento típico de nutriente de baixa mobilidade cujo teor se incrementa a medida em que a folha se torna mais velha (Figura 2B e 2C). Para o crescimento adequado do pinhão-manso esses nutrientes precisam ser continuamente absorvidos e translocados para as regiões em crescimento pois, em caso de deficiência, os sintomas surgiriam nas folhas mais jovens. 


\section{CONCLUSÕES}

1. Os nutrientes $\mathrm{P}, \mathrm{K}, \mathrm{Cu}$ e Zn são intensamente redistribuídos das folhas mais velhas para os tecidos mais jovens da planta de pinhão-manso enquanto ocorre pouca redistribuição de $\mathrm{N}, \mathrm{Ca}$, $\mathrm{Mg}, \mathrm{Fe}$ e Mn.

2. Com a senescência das folhas os nutrientes que não se redistribuem representam perda para a planta, de vez que precisam ser reabsorvidos para suportar o crescimento de novas estruturas.

\section{Agradecimentos}

Ao Banco do Nordeste (FUNDECI), FAPESQ/PB e ao CNPq, pelo apoio financeiro.

\section{LITERATURA CITADA}

Amaral, J. F. T. do.; Bruckner, C. H.; Martinez, H. E. P.; Cruz, C. D.; Godoy, C. L.; Caixeta, S. L. Determination of leaf sampling techniques to assess the nutritional status of barbados cherry (Malpighia emarginata D.C.). Fruits, v.57, p.161-171, 2002.

Arruda, F. P.; Beltrão, N. E. M.; Andrade, A. P.; Pereira, W. E.; Severino, L. S. Cultivo de pinhão manso (Jatropha curcas L.) como alternativa para o semiárido nordestino. Revista Brasileira de Oleaginosas e Fibrosas, v.8, p.789-799, 2004.

Blanchar, R.W.; Rehm, G.; Caldwell, A. C. Sulfur in plant material by digestion with nitric and perchloric acid. Proceedings of the Soil Science Society of America, v.29, p.71-72, 1963.

Braga, J. M.; Defelipo, B. V. Determinação espectrofotométrica de $\mathrm{P}$ em extratos de solo e material vegetal. Revista Ceres, v.21, p.73-85, 1974.

Camargos, S. L.; Muraoka, T. Teores, acúmulo e redistribuição de macronutrientes em castanheira-do-brasil. Revista Agricultura Tropical, v.10, p.72-83, 2007.

Caldeira, M. V. W.; Schumacher, M. V.; Rodrigues, L. M. Teor e redistribuição de nutrientes nas folhas e galhos em um povoamento de Acacia mearsii de Wild. (Acacia negra). Boletim de Pesquisa Florestal, v.45, p.69-88, 2002.

Epstein, E; Bloom, A. Nutrição mineral de plantas. 2.ed. Londrina: Planta, v.1, 2006. 416p.

George, A. P.; Nissen, R. J.; Carseldine, M. L. Effect of season (vegetative flushing) and leaf position on the leaf nutrient compositon of Annona spp. Hybrid cv. Pink's Mammoth in south-eastern Queensland. Australian Journal of Experimental Agriculture, v.29, p.587-595, 1989.
ITEP - Instituto de Tecnologia de Pernambuco. Médias históricas da chuva $(\mathrm{mm})$ de janeiro a dezembro para o Estado de Pernambuco. http://www.itep.br/meteorologia/ climatologia.htm. 02 Jun. 2008.

Kumar, P.; Pandey, R. M. Sampling for mineral content in leaves of guava cultivar 'Lucknow-49'. Scientia Horticulturae, v.11, p.163-174, 1979.

Laviola, B. G.; Dias, L. A. S. Teor e acúmulo de nutrientes em folhas e frutos de pinhão-manso. Revista Brasileira de Ciência do Solo, v.32, p.1969-1975, 2008.

Leitão, A. C.; Silva, O. A. Variação sazonal de macronutrientes em uma espécie arbórea de cerrado, na Reserva Biológica e Estação Experimental de Mogi-Guaçu, estado de São Paulo, Brasil. Rodriguésia, v.55, p.127-136, 2004.

Lima, R. L.; Severino, L. S.; Ferreira, G. B.; Sofiatti, V.; Sampaio, L. R.; Beltrão, N. E. de M. Casca de mamona associada a quatro fontes de matéria orgânica para a produção de mudas de pinhão-manso. Revista Ceres, v.58, p.232-237, 2011.

Lima, R. L.; Severino, L. S.; Pereira, W. E.; Lucena, A. M. A.; Gheyi, H. R.; Arriel, N. H. C. Comprimento das estacas e parte do ramo para formação de mudas de pinhão-manso. Revista Brasileira de Engenharia Agrícola e Ambiental, v.14, p.1234-1239, 2010.

Malavolta, E. Manual de nutrição mineral de plantas. São Paulo: Livroceres, 2006. 638p.

Marschner, H. Mineral nutrition of higher plants. London: Academic, 2002. 889p.

Medeiros, R. A.; Haridasan, M. Seasonal variations in the foliar concentrations of nutrients in some aluminium accumulating and nonaccumulating species of the cerrado region of central Brazil. Plant and Soil, v.88, p.433-436. 1985.

Pathar, R. A.; Pandey, R. M. Sampling for mineral content in leaves of mango cultivar 'Dashehari'. Scientia Horticulturae, v.5, p.255-264, 1976.

Santos, J. W.; Gheyi, H. R. Estatística experimental aplicada. Campina Grande: Gráfica Marcone, 2003.213p.

Villela, D. M.; Lacerda, L. D. Dinâmica de elementos minerais em folhas de duas espécies arbóreas de cerrado. Revista Brasileira de Biologia, v.52, p.151-160. 1992.

Yamada, T. Potássio: Dinâmica e disponibilidade no solo. In: Fernandes, F. M.; Nascimento, V. M. (ed.). Curso de atualização em fertilidade do solo. Campinas, Fundação Cargill, 1987, p.183-205. 